\title{
HALLAZGO DE DUNAS FÓSILES DE FINAL DEL PLEISTOCENO EN LAS ISLAS MURCIÉLAGO, COSTA RICA
}

\author{
Percy Denyer ${ }^{1 *}$, Jorge Cortés ${ }^{2} \&$ Guaria Cárdenes ${ }^{1}$ \\ ${ }^{1}$ Escuela Centroamericana de Geología, Universidad de Costa Rica, \\ Apdo. 214-2060 UCR, Costa Rica. \\ ${ }^{2}$ Centro de Investigación en Ciencias del Mar y Limnología (CIMAR), y Escuela de \\ Biología, Universidad de Costa Rica. \\ *Autor para contacto: pdenyer@geologia.ac.cr
}

(Recibido 25/01/06; aceptado 19/04/06)

\begin{abstract}
In this paper we described fossil dunes found at the Islas Murciélago, Área de Conservación Guanacaste, south of the Santa Elena Peninsula, on the northwest part of Costa Rica. Currently, this region is exposed to strong winds during the dry season (December to May), which produce coastal dunes. This process has been active at least since the Upper Pleistocene, as we propose with the finding of the fossil dunes in the area. These dunes have a maximum corrected age, based on a 14C radiometric dating, between 15981 and 17132 years before the present, which coincide with the Heinrich 1 event. This finding indicates a paleoclimate of the region as very dry and with very strong winds, related to the Heinrich 1 event after the Last Glacial Maximum, which was a dry period. The direction of the wind, based on the stratigraphy, was NE to SW and from the NNW to the SSE. These directions correspond with the present day winds. Key words: fossil dunes, paleoclimate, Heinrich 1, Costa Rica, Islas Murciélago.
\end{abstract}

RESUMEN: Se documenta la presencia de dunas fósiles en las islas Murciélago, ubicadas al S de la península de Santa Elena, en el NW de Costa Rica. Actualmente la región es afectada por fuertes vientos alisios durante la estación seca (diciembre-mayo), que en la actualidad son la causa de la existencia de dunas costeras. Este fenómeno ha estado activo, por lo menos desde el Pleistoceno Superior, como hemos podido comprobar con el hallazgo de dunas fósiles en la zona. Con base en una datación de radiométrica de ${ }^{14} \mathrm{C}$, se determina una edad entre 15,981 y 17,132 años antes del presente, que coincide con el evento Heinrich 1. Este hallazgo indica que, probablemente en esta región, hubo un paleoclima más seco, después de la última gran glaciación del final del Pleistoceno, con un clima más seco y con vientos de gran magnitud, cuya dirección fue de NE a SW y de NNW a SSE, basado en las estratificaciones medidas. Palabras clave: Dunas fósiles, paleoclima, Heinrich 1, Costa Rica, Islas Murciélago. 


\section{INTRODUCCIÓN}

La península de Santa Elena, ubicada en el noroeste de Costa Rica (Fig. 1), geológicamente está constituida por una secuencia de basaltos alcalinos, radiolaritas y brechas polimícticas, que forman el sustrato o autóctono relativo, de edad Jurásico-Cretácico, del nappe de Santa Elena, el cual está constituido por rocas máficas y ultramáficas, conocidas como las perdidotitas de Santa Elena (Harrison, 1953; Dengo, 1962; Tournon, 1984; 1994). Esta secuencia litológica está sobreyacida por una serie sedimentaria del Cretácico Superior hasta el Paleógeno (Dengo, 1962). Una cobertura ignimbrítica del del Neógeno y los depósitos coluvio-aluviales y costeros del Holoceno coronan la secuencia (Fig. 1).

En el extremo occidental de la península de Santa Elena y separadas $5 \mathrm{~km}$ de tierra firme se encuentran las islas Murciélago, que constituyen un archipiélago de 5 islas principales y por lo menos 10 islotes. La isla principal es conocida como San José (Fig. 2) y tiene $1,5 \mathrm{~km}^{2} \mathrm{de}$ área y una altura máxima de unos $140 \mathrm{~m}$. Las otras tienen un área menor que $1 \mathrm{~km}^{2}$ y alturas máximas de $83 \mathrm{~m}$ en la isla Catalina, $69 \mathrm{~m}$ en la isla Cocinero, $60 \mathrm{~m}$ de la isla Las Golondrinas y 42 en la isla San Pedrito. La morfología del fondo oceánico entre las islas y tierra firme es relativamente regular y plana, entre 60 y $80 \mathrm{~m}$ de profundidad, mostrando una pequeña plataforma sumergida a $20 \mathrm{~m}$ (Fig. 3). Geológicamente estas islas están constituidas por basaltos en columnas, almohadillas y masivos, su edad es de 109,0 \pm 2,0 millones de años (Hauff et al., 2000). Las islas de este archipiélago se alinean E-W, por una longitud de $8 \mathrm{~km}$, lo cual se debe a que la estructura de los basaltos que la conforman, tienen una inclinación subvertical hacia el norte (Fig. 2), por lo que su dirección estratigráfica es E-W. Dos islas cercanas, Colorada y Pelada, son geológicamente diferentes ya que son ultrabasitas del nappe de Santa Elena (Fig. 1).

La península de Santa Elena y la región aledaña es actualmente afectada por intensos vientos alisios, que llegan a esta región a causa de la depresión natural que corresponde con los lagos de Nicaragua y Managua. El viento

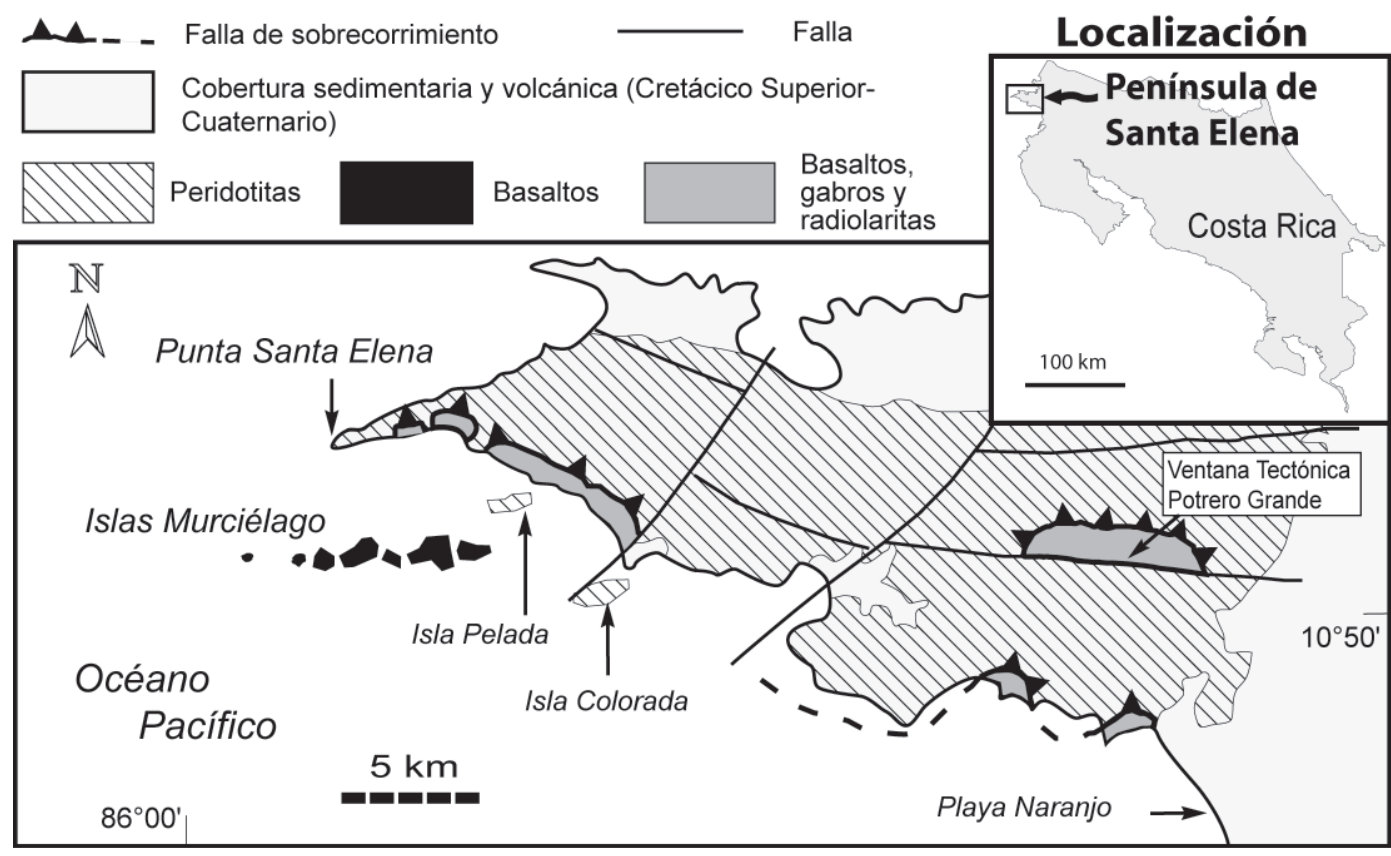

Fig. 1: Localización de la península de Santa Elena (Pen. Sta. Elena) y las islas Murciélago en su contexto geológico generalizado. 


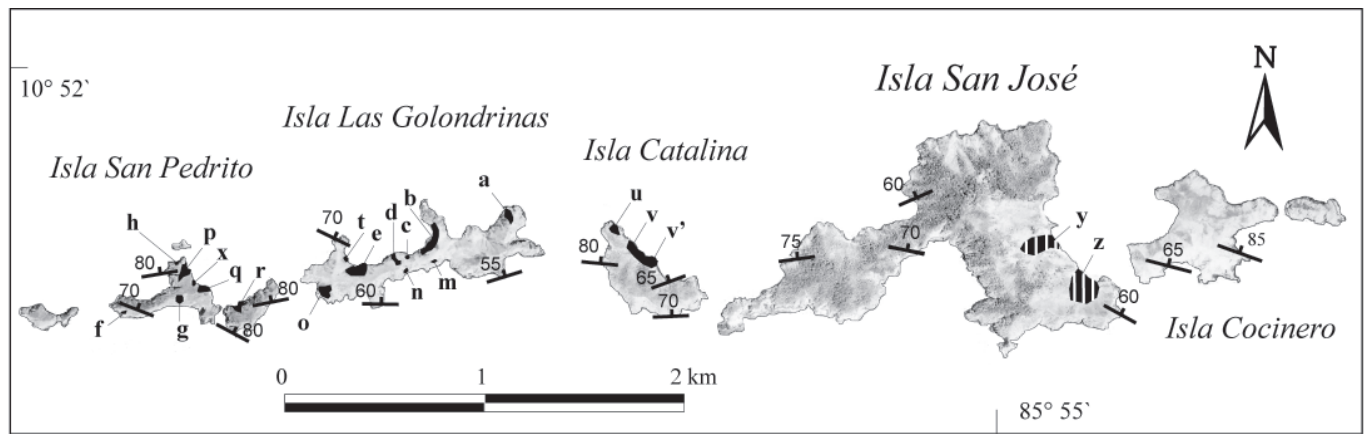

Fig. 2: Ubicación de las dunas (en negro), las dunas fósiles están codificadas de la "a" a la "v" y las dunas recientes (en negro con líneas blancas verticales) están señaladas como "y", "z". Los símbolos de dirección estratigráfica y buzamiento corresponden a las estimaciones hechas con las pillows y las columnas de los basaltos que conforman las islas Murciélago. La textura de las islas corresponde con fotografias aéreas del I.G.N 14248, 14249, 14250 y 14251 escala 1:20 000.

pasa libremente a través de Centroamérica (Banichevich \& Castro, 1999), con una dirección de NE a SW. Esto causa que durante los meses de la estación seca, diciembre-mayo, haya un intenso oleaje y disminución de la temperatura del agua marina hasta unos $14^{\circ} \mathrm{C}$, por la alteración de la termoclina (Fiedler, 2002). Estos vientos desplazan las aguas marinas superficiales dándose los fenómenos de upwelling, o sea la consecuente "surgencia" de aguas frías del fondo cargadas con nutrientes. La fuerza del viento tiene una magnitud máxima de $10^{-8}$ dinas ${ }^{*} \mathrm{~cm}^{-3}$ (Hofmann et al., 1981). Estas condiciones climáticas particulares han causado la formación de dunas costeras en el NW de Costa Rica.
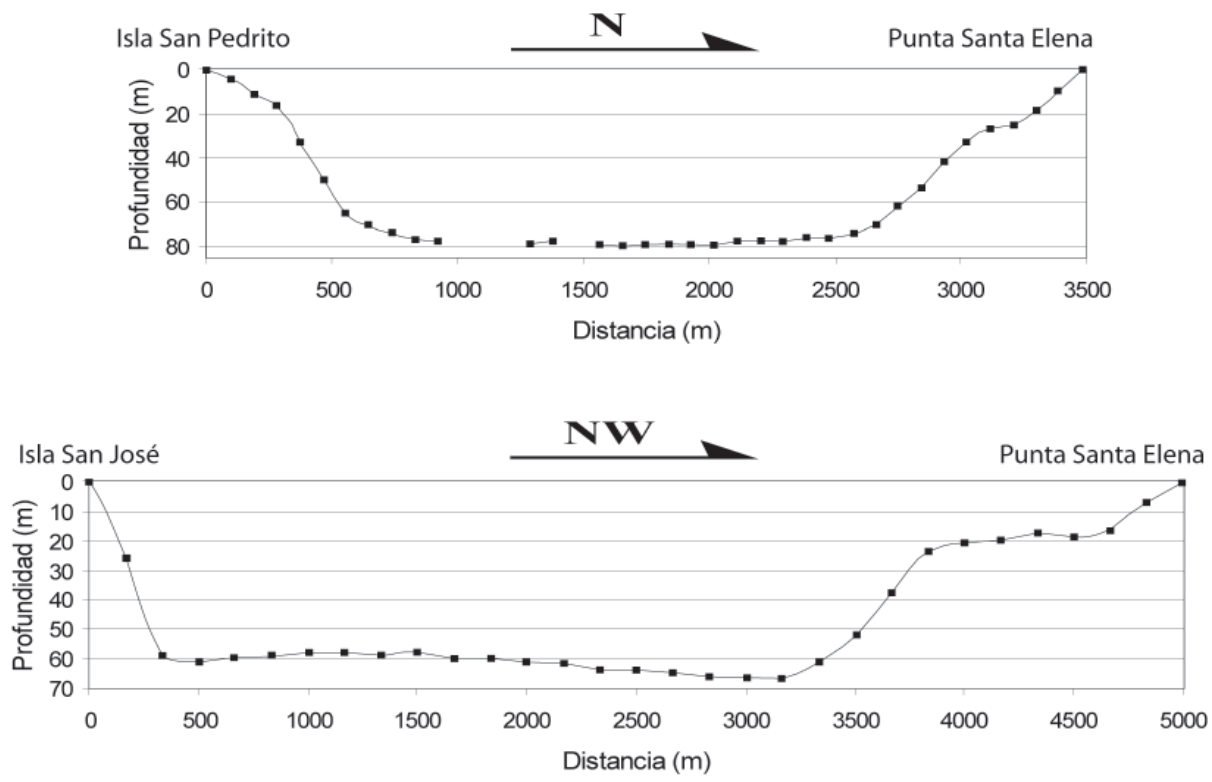

Fig. 3: Perfiles batimétricos entre las islas Murciélago y punta Santa Elena. 
En este trabajo se documenta la presencia de dunas fósiles y recientes que se formaron en la zona costera del flanco norte de las islas Murciélago y presentamos información sobre su morfología, composición, flora, posible origen y edad, así mismo analizamos las implicaciones que se derivan de las mismas.

\section{MÉTODOS}

Se hizo un cartografiado de las diferentes dunas desde un bote inflable y se escogieron algunos puntos estratégicos para hacer observaciones directas y un mapeo de detalle con brújula y cinta. Se tomaron los datos de la estructuración interna de las dunas, los cuales se procesaron en la red estereográfica, utilizando el programa Tectonics FP, versión 1.26.1152-Dem (Reiter \& Acs, 2002).

Para conocer de la morfología del fondo marino entre tierra firme y las islas se hicieron dos perfiles batimétricos, uno con dirección NW-SE desde punta Santa Elena a la isla San José y otro $\mathrm{N}-\mathrm{S}$ de la misma punta hasta la isla San Pedrito.

En cada uno de estos perfiles se tomaron medidas a intervalos regulares de tiemwpo con una ecosonda manual de medida única, dichos datos luego fueron graficados (Fig. 3).

Se hizo un muestreo de los materiales que forman las dunas tanto fósiles como recientes, se tomaron alrededor de 30 muestras en los bordes costeros erosionados, de las cuales se procesaron las 20 muestras más representativas. Se elaboraron secciones delgadas de las muestras recolectadas y se estudiaron con el microscopio petrográfico para determinar su composición y granulometría. La granulometría se realizó por medio de mediciones del diámetro de los fragmentos, usando el micrómetro del microscopio en una cuadrícula marcada previamente en cada sección delgada; las medidas milimétricas se convirtieron en unidades $\varphi$ y se procesaron en una hoja electrónica diseñada en EXCEL, donde se obtuvieron los gráficos.

Para poder comparar el sedimento de las paleodunas con el de las dunas activas de la isla San José (Fig. 2) se tomaron muestras en trincheras excavadas de $1 \mathrm{~m}$ de hondo, y se muestreó cada $25 \mathrm{~cm}$ de profundidad. También se procesó una muestra de la arena de la playa en la isla San José (Fig. 2). Con las muestras de sedimentos sueltos se hicieron pastillas con resina, para hacer secciones delgadas y se procedió de la

Cuadro 1

Medida de las estratificaciones cruzadas de las dunas fósiles

\begin{tabular}{|c|c|c|c|c|c|c|c|c|c|c|c|}
\hline$D$ & D.I. & A.I. & $D$ & D.I. & A.I. & $D$ & D.B. & A.I. & $D$ & D.I. & A.I. \\
\hline$a$ & 180 & 20 & $\mathrm{~b}$ & 190 & 10 & $q$ & 328 & 32 & $q$ & 320 & 23 \\
\hline b & 220 & 15 & $\mathrm{C}$ & 330 & 15 & $q$ & 330 & 28 & $q$ & 325 & 27 \\
\hline b & 260 & 15 & $d$ & 350 & 10 & $q$ & 338 & 28 & $q$ & 320 & 48 \\
\hline b & 220 & 10 & $\mathrm{~d}$ & 230 & 25 & $q$ & 332 & 34 & $q$ & 260 & 20 \\
\hline b & 245 & 20 & $\mathrm{e}$ & 10 & 20 & $q$ & 324 & 28 & $q$ & 340 & 50 \\
\hline b & 245 & 17 & $\mathrm{e}$ & 280 & 20 & $q$ & 315 & 25 & $r$ & 320 & 15 \\
\hline b & 255 & 15 & $\mathrm{e}$ & 340 & 22 & $q$ & 320 & 23 & $\mathrm{u}$ & 220 & 20 \\
\hline b & 4 & 20 & $\mathrm{~g}$ & 170 & 17 & $q$ & 325 & 27 & $\mathrm{v}$ & 340 & 25 \\
\hline b & 245 & 24 & $\mathrm{~h}$ & 270 & 20 & $q$ & 320 & 48 & $\mathrm{v}$ & 150 & 30 \\
\hline b & 80 & 17 & $p$ & 350 & 20 & $q$ & 250 & 20 & $v^{\prime}$ & 340 & 25 \\
\hline b & 260 & 15 & $q$ & 230 & 30 & $q$ & 340 & 50 & $v^{\prime}$ & 90 & 25 \\
\hline b & 359 & 18 & $q$ & 33 & 15 & $q$ & 315 & 25 & $x$ & 260 & 30 \\
\hline b & 190 & 15 & $q$ & 185 & 3 & & & & & & \\
\hline
\end{tabular}

D: duna ubicada en figuraww 2, D.I.: dirección de inclinación, A.I.: ángulo de inclinación 


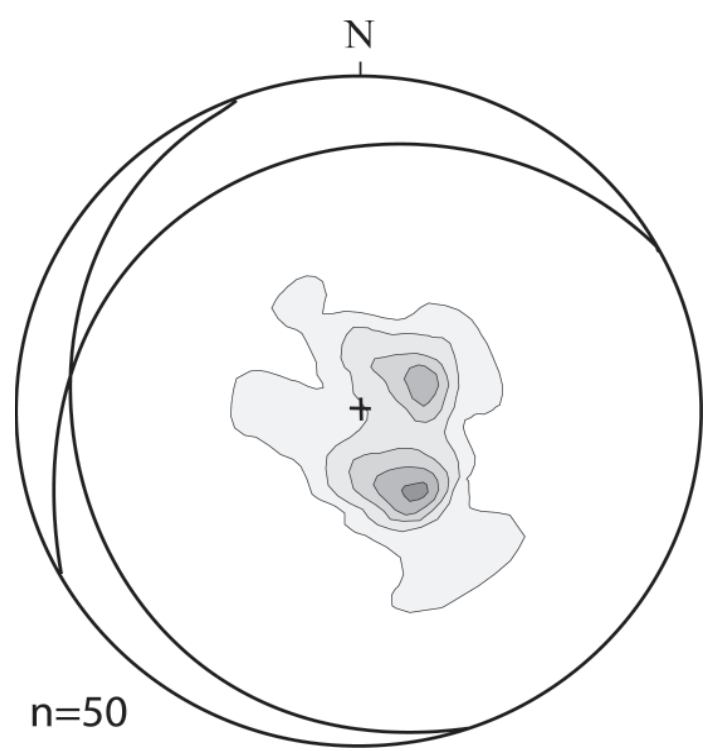

Fig. 4: Estadística en la red de Schmidt de las estratificaciones de las dunas fósiles, mostrando los planos de los 2 máximos. Se utilizó el programa TectonicsFP. Los contornos están en 1, $4,8,12$ y 16 por $1 \%$ de unidad de área.

misma manera que con las muestras de las dunas fósiles.

Se hizo una datación radiométrica de una muestra (Muestra 318A) de la isla San Pedrito ("q", Fig. 2), esta muestra se escogió debido a que los fragmentos de arena son relativamente más grandes. La muestra se desintegró físicamente y se separaron los granos. La datación se hizo con radiocarbono en el laboratorio Beta Analytic Inc. en noviembre de 1995 y así se obtuvo una edad de ${ }^{14} \mathrm{C}$ convencional en años BP (antes del presente). Debido a la naturaleza arenosa de la muestra se aplicó en el laboratorio, la técnica de tiempo de conteo extendido. Este dato se calibró usando las bases de datos de calibración de Hughen et al. (2004), y se utilizó el programa CALIB 5,0 Stuiver et al. (2005), basado en Stuiver \& Reimer (1993), programa desarrollado por el Quaternary Isotope Lab. de la Universidad de Washington. La calibración se hizo para los extremos mínimo y máximo de sigma 1 (68\% de probabilidad) y para sigma $2(95 \%$ de probabilidad).

\section{DUNAS FÓSILES}

Las dunas fósiles se presentan en las islas Catalina, Las Golondrinas y San Pedrito (Fig. 2). Las mejores exposiciones están en las laderas del sector norte de las islas, aunque en algunos casos el sedimento arenoso llegó a sobrepasar la divisoria de aguas, lo que propició la formación de dunas también en el sector sur. La parte frontal de las dunas fósiles se encuentran en la línea de costa, lo que permite actualmente la erosión de las mismas por el oleaje y las corrientes marinas. Por esta razón y por presencia de cobertura vegetal en algunos casos no fue posible establecer sus dimensiones.

En cuanto a su estructura interna las dunas presentan como rasgo sobresaliente estratificación cruzada decimétrica planar, con ángulos que alcanzan hasta $50^{\circ}$. Se tomaron 50 datos de las estratificaciones cruzadas, en las diferentes dunas (Cuadro 1), las cuales se graficaron en la red estereográfica (Fig. 4), donde se observan dos máximos estadísticos muy claros, $330 / 24^{\circ} \mathrm{y}$ $249 / 16^{\circ}$.

Todas las dunas presentan cementación de carbonato de calcio el cual pudo ser incorporado a las dunas por medio del splash marino y por la disolución de alguna porción de los fragmentos bioclásticos, que en la mayoría de los casos sobrepasan el $50 \%$ del sedimento.

La diferencia litológica entre las dunas y los basaltos que afloran en las islas causa diferencias

Cuadro 2

Dimensiones de las dunas de la isla Las Golondrinas

\begin{tabular}{cccc}
\hline $\begin{array}{c}\text { Dunas } \\
\text { isla Las } \\
\text { Golondrinas }\end{array}$ & $\begin{array}{c}\text { Largo } \\
(\mathrm{m})\end{array}$ & $\begin{array}{c}\text { Ancho } \\
(\mathrm{m})\end{array}$ & $\begin{array}{c}\text { Espesor } \\
\text { observado (m) }\end{array}$ \\
\hline $\mathrm{a}$ & 20 & - & 8 \\
$\mathrm{~b}$ & 50 & - & 20 \\
$\mathrm{c}$ & 10 & 10 & 5 \\
$\mathrm{~d}$ & 30 & - & 5 \\
$\mathrm{e}$ & 50 & 40 & 15 \\
$\mathrm{~m}$ & 15 & 15 & 10 \\
$\mathrm{n}$ & 15 & - & 10 \\
$\mathrm{o}$ & 30 & - & 8 \\
\hline
\end{tabular}




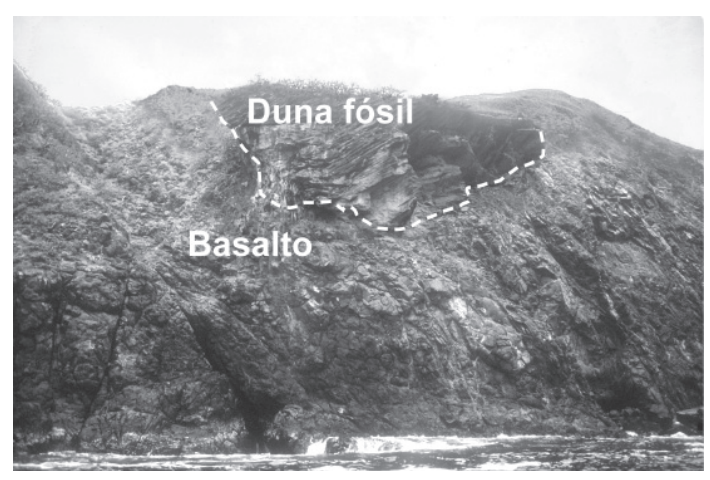

Fig. 5: Duna fósil colgada (la línea punteada indica el contorno de la misma), como relicto de la erosión. Isla Las Golondrinas (duna "a", Fig. 2)

en la vegetación que crece actualmente. Sobre las dunas fósiles crecen arbustos de la especie, Amphipterygium adstringens, la cual se ha encontrado en Costa Rica solamente en las islas Murciélago (J. Gómez Laurito, com. pers. 2002). El resto de las islas, constituido por basaltos, está cubierto por varias especies de pastos (Dauphin \& Grayum, 2005). Por lo tanto existe una clara correlación entre el sustrato litológico y la vegetación que se desarrolla sobre este.

\section{Dunas en isla Las Golondrinas}

Dimensiones $\boldsymbol{y}$ forma: En esta isla se presentan 9 dunas, tres se localizan en el sector sur y las restantes en el norte (Fig. 2), presentan dimensiones variables, no sobrepasando los 50 $\mathrm{m}$ de largo (Cuadro 2). En algunos casos solo es posible observar relictos de dunas que tuvieron mayor tamaño. Las dunas "a" (Fig. 5) y "o" son de tipo "colgado", es decir que son relictos de erosión que están a más de $5 \mathrm{~m}$ sobre el nivel del mar actual, por lo que hoy en día no están expuestas a la erosión marina. Debido a la erosión costera a la que han estado sometidas no es posible realizar una clasificación morfológica de ellas.

Aspectos granulométricos: Los sedimentos de todas las dunas presentan tamaños de grano que oscilan entre -1 y $4,27 \varphi$, siendo las fracciones arenosas las predominantes. Las fracciones de grava fina $(-1$ a $-0,50 \varphi)$ constituyen menos del $3 \%$ en la duna "t" (Fig. 6), estando presente solo en esta. Los tamaños limo-arcillosos en todas las dunas constituyen menos del $2 \%$ de los sedimentos. La mediana (D 50, según Trask, 1930) se encuentra entre 1,23 y $2 \varphi$, lo cual corresponde con arena media, la moda $(\mathrm{M}=$ punto de mayor altura en la curva, según Trask (1930) varía entre 0,23 y $2,74 \varphi$. La tendencia de las curvas es hacia la asimetría negativa, con comportamientos desde unimodales hasta polimodales. Se pueden observar las curvas acumulativas que nos dejan entre ver la regular selección de los materiales (Fig. 6), los cuales se pueden clasificar desde el punto de vista del tamaño de grano como arenas medias bien seleccionadas.

Composición de los granos: El principal material que compone los sedimentos de las dunas fósiles de isla Las Golondrinas son fragmentos bioclásticos, producidos por la erosión y transporte marino y eólico de esqueletos de bivalvos y gastrópodos, entre otros (Fig. 7). Los bioclastos presentan una baja esfericidad y son subredondeados (Fig. 8A), lo que implica relaciones esfericidad/redondez $(\mathrm{E} / \mathrm{R})$ entre 0,1 y 0,3 ; en algunas ocasiones se presenta orientación/ imbricación de los fragmentos. Los otros materiales corresponden con fragmentos de lutitas, peridotitas y de rocas ígneas sin diferenciar, estas últimas son las más abundantes. El contacto entre granos es en su gran mayoría puntual, y a veces flotante (Fig. 8B). Los sedimentos se encuentran

Cuadro 3

Dimensiones de las dunas de la isla San Pedrito

\begin{tabular}{cccc}
\hline $\begin{array}{c}\text { Dunas isla } \\
\text { San Pedrito }\end{array}$ & $\begin{array}{c}\text { Largo } \\
(\mathrm{m})\end{array}$ & Ancho $(\mathrm{m})$ & $\begin{array}{c}\text { Espesor } \\
\text { observado } \\
(\mathrm{m})\end{array}$ \\
\hline $\mathrm{g}$ & 10 & - & 7 \\
$\mathrm{~h}$ & 35 & - & 15 \\
$\mathrm{p}$ & 75 & - & 8 \\
$\mathrm{q}$ & 60 & 20 & 11,5 \\
$\mathrm{r}$ & 10 & - & - \\
$\mathrm{x}$ & 20 & 20 & - \\
\hline
\end{tabular}



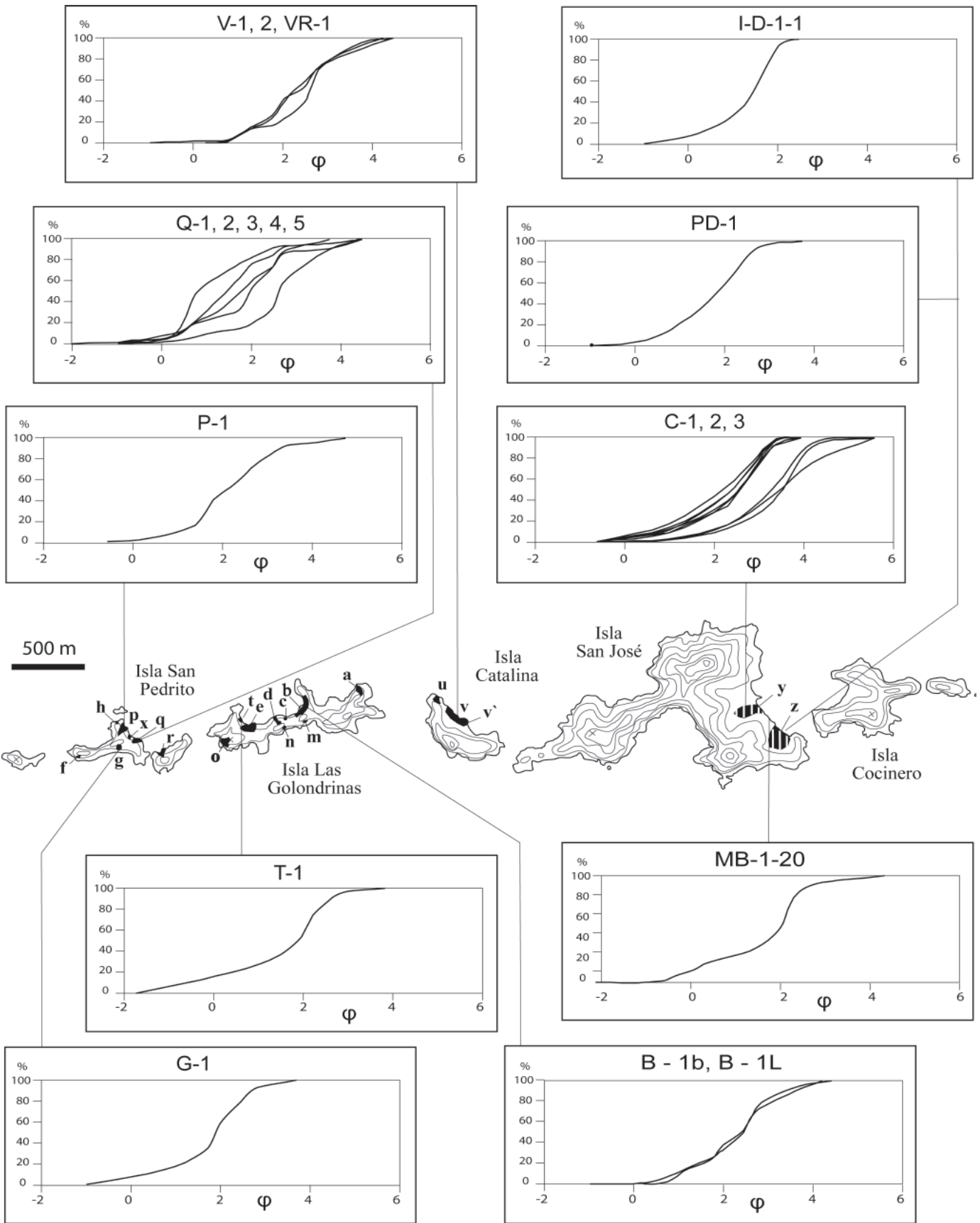

Fig. 6: Análisis granulométricos las dunas fósiles, las activas y la arena de la playa de la isla San José. La codificación de la parte superior de los gráficos corresponde con las muestras analizadas. El eje de x corresponde con unidades $\varphi$. Las dunas fósiles se muestran en negro y las recientes en negro con líneas blancas verticales; ambas se nombran con letras. Las curvas de nivel mostradas son cada $20 \mathrm{~m}$, basados en la cartografía del I.G.N. y el límite litoral se trazó con base en fotografías aéreas que se muestran en la figura 2. 

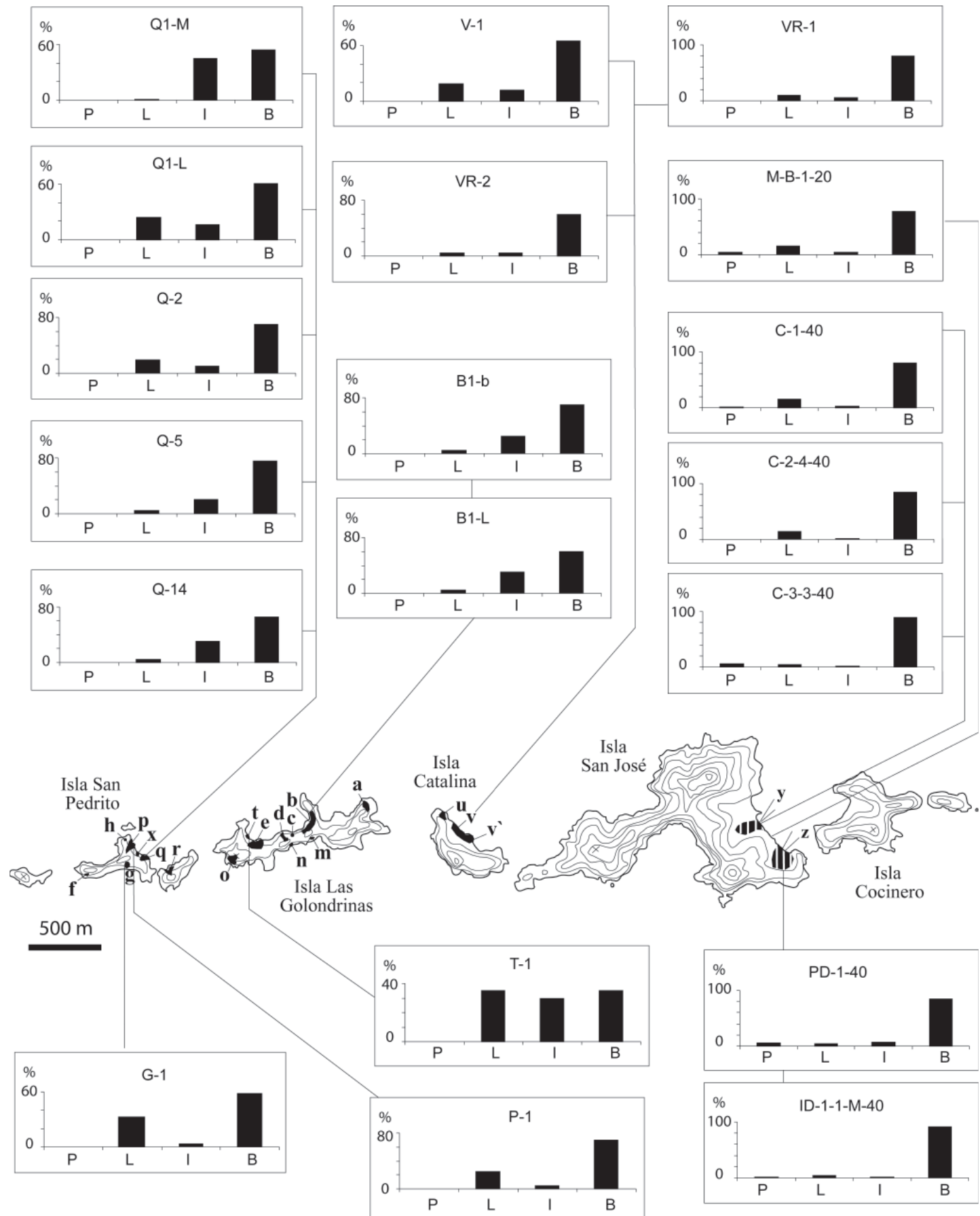

Fig. 7: Composición litológica de los granos de arena que forman las dunas fósiles, las activas y la arena de la playa de la isla San José. $\mathrm{P}=$ peridotitas, $\mathrm{L}=$ lutitas, $\mathrm{I}=$ rocas ígneas y $\mathrm{B}=$ bioclastos y calcita. Las dunas fósiles se muestran en negro y las recientes en negro con líneas blancas verticales; ambas se nombran con letras. Las curvas de nivel mostradas son cada 20 m, basados en la cartografía del I.G.N. y el límite litoral se trazó con base en fotografías aéreas que se muestran en la Fig. 2. 

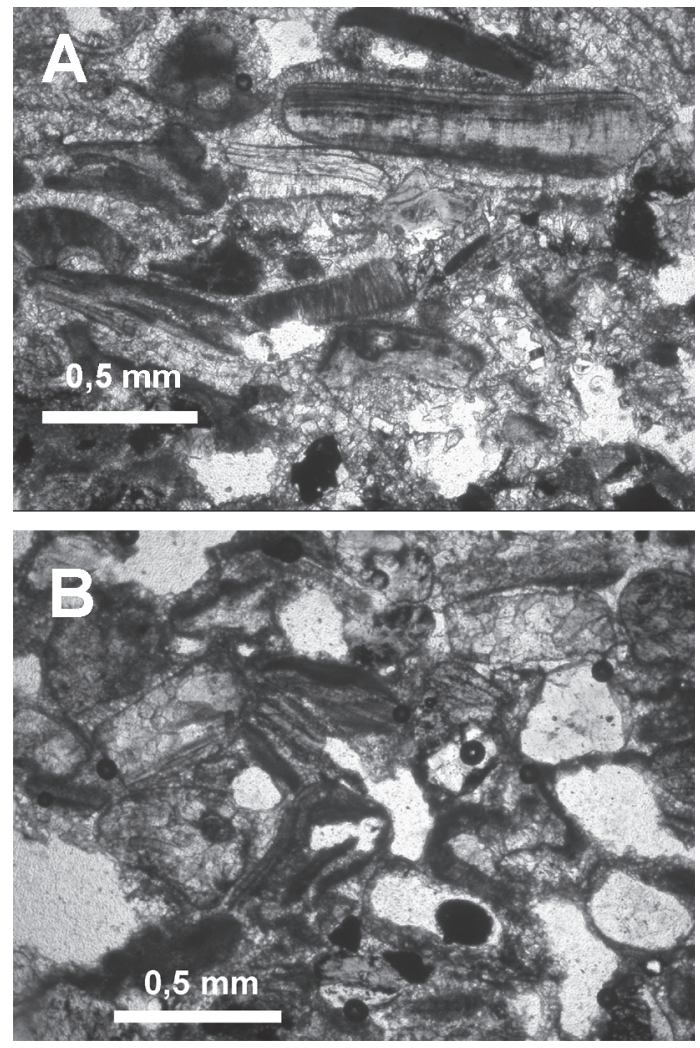

Fig. 8: A) Microfotografía de la duna "b" (muestra B-1-b) en isla Las Golondrinas, se notan los bioclastos en contacto puntual con cemento esparítico. B) Microfotografía de la duna " $b$ " (muestra B-1-L) donde se muestra la redondez de los clastos y el contacto puntual.

unidos por la presencia de cemento esparítico (Fig. 8A). Por lo anterior las areniscas que forman el complejo de dunas de isla Las Golondrinas se puede considerar como una Bioesparita (Folk, 1962) (ver composición en Fig. 7).

\section{Dunas en isla San Pedrito}

Dimensiones, forma y estructura interna: En San Pedrito se ubicaron 2 dunas en el sector sur ("f" y "g", Fig. 2)y 5 en el norte ("h", "p", "x", "q" y "r", Fig. 2). Al igual que en la isla Las Golondrinas estas presentan tamaños variable, siendo la máxima de $75 \mathrm{~m}$ de largo (Cuadro 3). La duna "f" está colgada a 20 m aproximadamente sobre el nivel del mar. La duna "q" se presenta como una estructura a la orilla del mar ("q", Fig. 2; Fig. 9) y tiene estratificaciones cruzadas muy destacantes (Fig. 10).

Aspectos granulométricos: Los materiales tienen tamaños, que van desde $-1 \quad \varphi$ hasta fracciones menores a los 4,25 $\varphi$ (Fig. 6). La fracción de grava fina solo se presenta en la duna P y alcanza el $3 \%$; mientras que los limos y arcillas en todas las dunas no sobrepasan el $5 \%$, el porcentaje restante corresponde con materiales arenosos desde finos hasta gruesos. La mediana varía entre los $1,23 \varphi$ y $2,74 \varphi$, o sea entre arena media a fina. Los materiales son polimodales correspondiendo con los tamaños 1,74, 2,47 y $3,74 \varphi$. La asimetría de las curvas es negativa a neutra (algunos sectores de la duna "q") y tienen comportamientos unimodales en su mayoría, aunque también es posible encontrar curvas bimodales y polimodales. Por medio de la figura 6 se muestra como la selección de los materiales es regular. Desde el punto de vista granulométrico se puede considerar como una arena media.

Composición de los granos: Al igual que en la isla Las Golondrinas, en San Pedrito la principal fuente de sedimento de las dunas son los bioclastos con porcentajes entre 55 y $70 \%$, los cuales son fragmentos producto de la erosión de esqueletos de bivalvos, gastrópodos y algas (Fig. 7). Los bioclastos en cuanto a su forma son sub redondeados de baja esfericidad, son relaciones esfericidad redondez (E/R) entre 0,5 y 0,7 . Los bioclastos de la muestra tomada en la duna "p" presentan micritización. Los fragmentos de

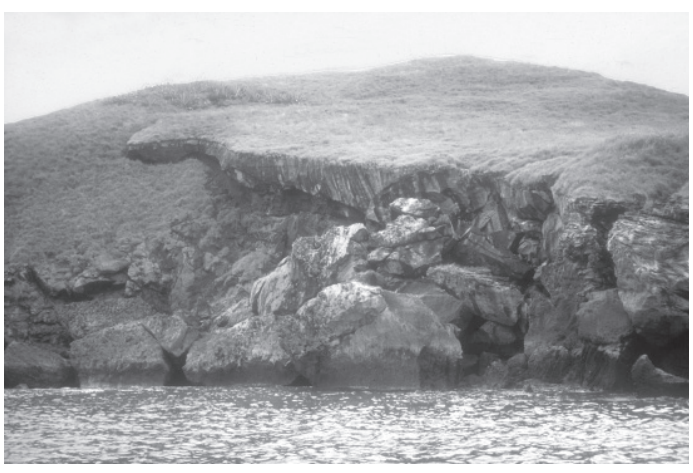

Fig. 9: Duna fósil de la isla San Pedrito (“q”, Fig. 2). 
Cuadro 4

Dimensiones de las dunas de la isla Catalina

\begin{tabular}{ccc}
\hline $\begin{array}{c}\text { Dunas isla } \\
\text { Catalina }\end{array}$ & Largo (m) & Ancho (m) \\
\hline $\mathrm{u}$ & 10 & - \\
$\mathrm{v}$ & 75 & - \\
$\mathrm{v}^{\prime}$ & 15 & 15 \\
\hline
\end{tabular}

lutitas, peridotitas y rocas ígneas sin diferenciar presentan fuertes variaciones de porcentaje de una muestra a la otra, inclusive dentro de una misma duna (p.e. duna “q”); son sub-angulosos y de baja esfericidad, con relaciones E/R de 0,1. En muchas de las muestras colectadas de las dunas de la isla San Pedrito se encontraron zonas de acumulación centimétricas de hematita, producto de la meteorización de los fragmentos de las rocas tanto ígneas como de las peridotitas. El contacto entre los granos es puntual concavo-convexo, en algunos niveles de la duna "q", lo que indica un empaquetamiento muy bueno. El cemento es esparítico, y las rocas se pueden clasificar en general como Bioesparitas (Folk, 1962).

Edad: La muestra 318A (muestra Beta-86182 en el laboratorio Beta Analytic) dio una edad de ${ }^{14} \mathrm{C}$ convencional de $14300 \pm 180 \mathrm{BP}$, que ya calibrado implica que los límites de edad varían de 16261 a 16848 años BP (sigma 1) y entre 15 981 y 17132 años BP (sigma 2) (Fig. 11).

\section{Dunas en isla Catalina}

Dimensiones y forma: Se presentan 3 dunas en el sector noreste, la más desarrollada presenta un largo de $75 \mathrm{~m}$; en el Cuadro 4 se presentan sus dimensiones.

Aspectos granulométricos: Los materiales que forman estas dunas tienen tamaños que van desde $0,74 \varphi$ hasta menores a 4,27 $\varphi$. La fracción de limos y arcillas representa menos del 3\% del material, no se da la presencia de materiales con tamaños superiores o iguales a las gravas finas. La mediana está conformada por sedimentos entre 1,74 y $2 \varphi$, las modas se presentan en los tamaños $1,74,2,47$ y 3,74 $\varphi$. La asimetría es negativa y los comportamientos de las curvas pueden ser desde unimodales hasta polimodales (Fig. 6). La selección de los sedimentos es de regular a mala. Los sedimentos que forman estas dunas son arenas medias.

Composición de los granos: Los bioclastos son los materiales dominantes en las dunas de isla Catalina (Fig. 7), estos constituyen entre el 55 y $70 \%$, estos son redondeados de baja esfericidad, con índices $\mathrm{E} / \mathrm{R}$ de 0,5 . Los otros fragmentos líticos son de lutitas, rocas ígneas y en menor proporción de peridotitas, estos tienen una muy baja esfericidad y son sub-redondeados, $\mathrm{E} / \mathrm{R}$ de 0,1 . El contacto entre los granos es puntual, lo que implica un buen empaquetamiento, observable bajo el microscopio. En algunos casos se presenta micro-alineación y micro-imbricación a nivel microscópico. En cuanto a su clasificación, estas

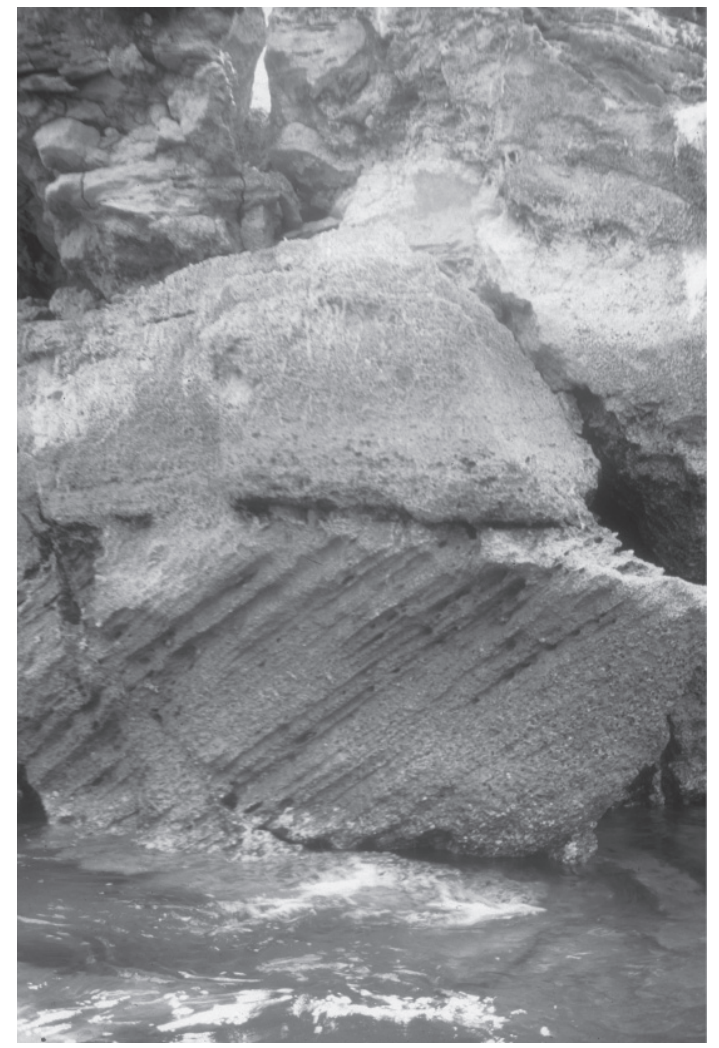

Fig. 10: Estratificación cruzada decimétrica en la isla San Pedrito (“q", Fig. 2). 


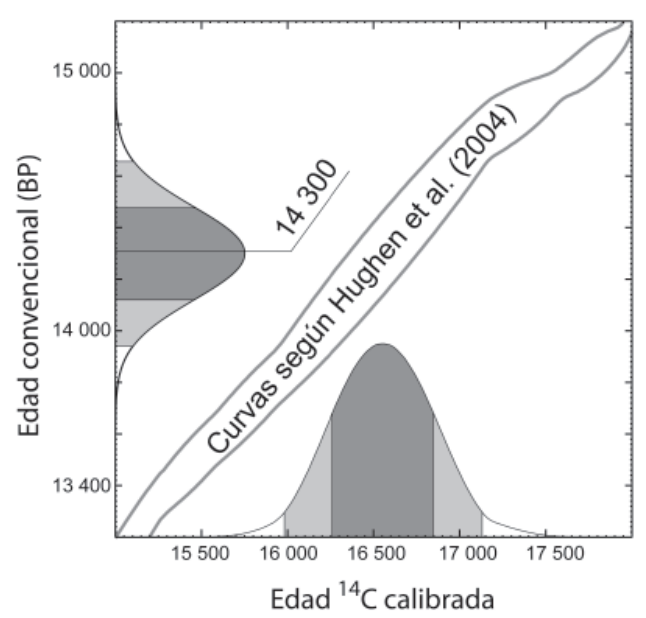

Fig. 11: Calibración de la muestra 318A, usando la base de datos marine 04.14c (Hughen et al., 2004) y el programa CALIB 5 (Stuiver et al., 2005). El gris más oscuro es la probabilidad sigma 1 y el más claro es la probabilidad sigma 2 .

rocas son, en su mayoría, Biomicritas y una muestra de la duna "v" es una Bioesparita (Folk, 1962).

Otros elementos biogénicos presentes: Se encontraron gran cantidad de moldes de raíces, ahora parcialmente meteorizadas (Fig. 13). Estas raíces fueron identificadas como pertenecientes al género Dalbergia (Papillionacea: Leguminosa). Hay varias especies de este género en el Pacífico de Costa Rica. Es un arbusto abundante en la zona costera norte. Se midió el diámetro de 126 ejemplares, aflorando en un área de 2 $\mathrm{m}^{2}$, lo que dio un promedio de diámetro entre 0,5-2,0 cm, y un máximo de $10 \mathrm{~cm}$ (Fig. 14). Valga decir que una vegetación muy similar, Dalbergia chontalensis (conocida anteriormente como D. glabra), actualmente es abundante en Isla Cocinero y en tierra firme en la Península de Santa Elena (M. Grayum, com. pers. 2006).

\section{Dunas activas de isla San José}

Dimensiones y forma: Solo se observaron dunas costeras activas en la isla San José, aunque valga destacar que en los alrededores continentales de las islas Murciélago existen varios sitios con dunas activas. Existen dos dunas principales separadas por un acantilado de basalto (Fig. 2). La duna " $z$ " al SSE tiene $70 \mathrm{~m}$ de ancho en la dirección NE-SW y un largo de $150 \mathrm{~m}$ en la dirección NW-SE, paralelo a la línea de playa, tiene un alto máximo de $8,5 \mathrm{~m}$ y en su parte superior crecen algunos pastos y árboles de jocote. La duna y al NNW tiene $75 \mathrm{~m}$ de largo en una dirección NE-SW y un ancho de $25 \mathrm{~m}$ y su altura máxima es de $11,5 \mathrm{~m}$; solo tiene crecimiento de pastos encima (Fig. 15).

Aspectos granulométricos: Todas las muestras de las dunas reciente tienen un rango granulométrico entre 0,23 y menores a $4,25 \varphi$, lo que implica que se trata de arenas de gruesas a finas, la fracciones finas no sobre pasan el $10 \%$. La mediana es en todos $\operatorname{los} \operatorname{casos} 1,23 \varphi$ y la moda también corresponde con 1,23 $\varphi$. La asimetría de las curvas en su mayoría neutra, aunque a veces es ligeramente negativa, ya que tiende a presentar un alto porcentaje de arenas fina, son curvas claramente unimodales (Fig. 6). En cuanto a la selección es muy buena.

Composición de los granos: Los materiales que componen las dunas recientes son prácticamente bioclásticos, al contabilizar los otros tipos de materiales (lutitas, perodotitas y rocas ígneas sin diferenciar) estos no alcanzan

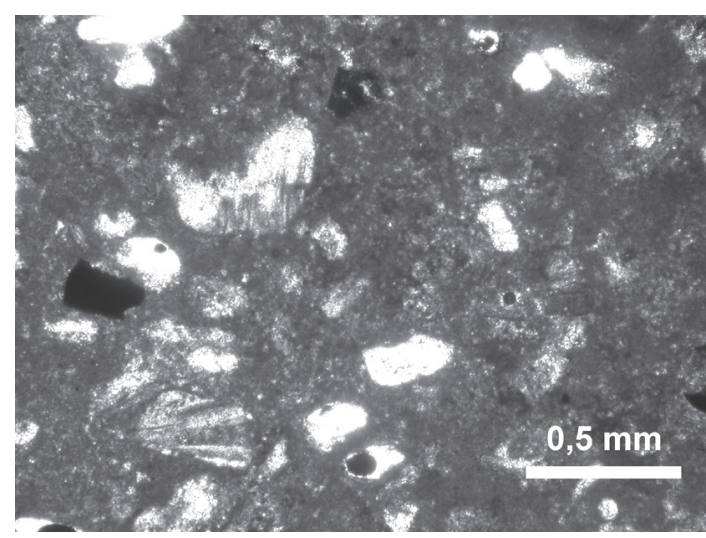

Fig. 12: Microfotografía de la duna "v" en la isla Catalina (muestra v-r-2), se observa el cemento micrítico y los bioclastos micritizados. 


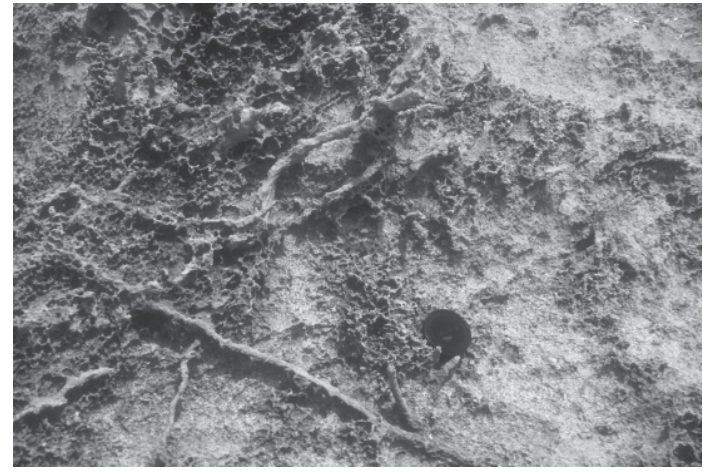

Fig. 13: Moldes positivos de las raíces encontrados en las dunas fósiles de la isla Catalina ("v", Fig. 2). La tapa de lente fotográfico tiene $52 \mathrm{~mm}$ de diámetro.

el 5\% (Fig. 7). Los bioclastos que se presentan son fragmentos de algas, gastrópodos, bivalvos, y corales, entre otros, son redondeados y subesféricos, con índices E/R de hasta 0,9.

Elementos biogénicos: Las dunas actuales están cubiertas por árboles de jocote y por algunos pastos (principalmente $J$. pilosa), posiblemente de la especie Jouvea pilosa. Esta última especie se encuentra comúnmente creciendo sobre arenas en las costas (M. Grayum, com. pers. 2006).

\section{SEDIMENTOS DE PLAYA}

Además de las dunas activas se muestreó el material de la zona intermareal de la playa en la isla San José, con el fin de hacer una comparación entre este y el material tanto de las dunas activas como el de las fósiles.

Aspectos granulométricos: Los materiales de la playa en isla San José tienen tamaños que van desde $-1 \varphi$ hasta $4,27 \varphi$, las gravas finas se presentan con porcentajes menores al $4 \%$ mientras que los limos y arcilla equivalen a menos del $8 \%$. Los materiales predominantes son arenas de gruesas a finas. La mediana se encuentra en tamaños de $2 \varphi$, y la moda presenta dos valores $0,23 \varphi$ y $2,23 \varphi$. La asimetría es ligeramente negativa, con curvas de comportamiento bimodal (Fig. 7).
Composición de los granos: Al igual que en las dunas activas los bioclastos son los materiales que componen mayoritariamente las arenas de playa (Fig. 7). Estos son redondeados y tiene baja esfericidad, con relaciones $\mathrm{E} / \mathrm{R}$ entre 0,3 y 0,5 . Las peridotitas, lutitas y rocas ígneas no diferenciadas en conjunto no sobrepasan el 15\%. De la misma manera que los bioclastos estos materiales son redondeados y tienen baja esfericidad.

\section{COMENTARIOS Y DISCUSIÓN}

Los sedimentos que forman las dunas fósiles, las dunas actuales y las playas son arenas de gruesas a finas, con escaso contenido de grava fina, limo y arcilla. Esta buena selección implica que la acción erosiva y de transporte marino y eólico ha sido eficiente desde el pasado reciente. En el cuadro 5 se presenta una comparación de las dunas representativas de cada una de las islas. Los materiales que forman tanto las dunas fósiles, las actuales y los sedimentos de playa varían entre -1 y $4,2 \varphi$. La mediana tanto de los materiales litificados como de los sedimentos no consolidados oscila entre 1,2 y $2,7 \varphi$, lo que corresponde con arenas medias a finas. La moda presenta dos tendencias, una unimodal con valores que van desde 1,2 hasta $2,2 \varphi$ para la playa y las dunas actuales, y la otra polimodal, presente en casi todas las dunas fósiles, con valores que van de 1,7 a 3,7 $\varphi$ (Cuadro 5).

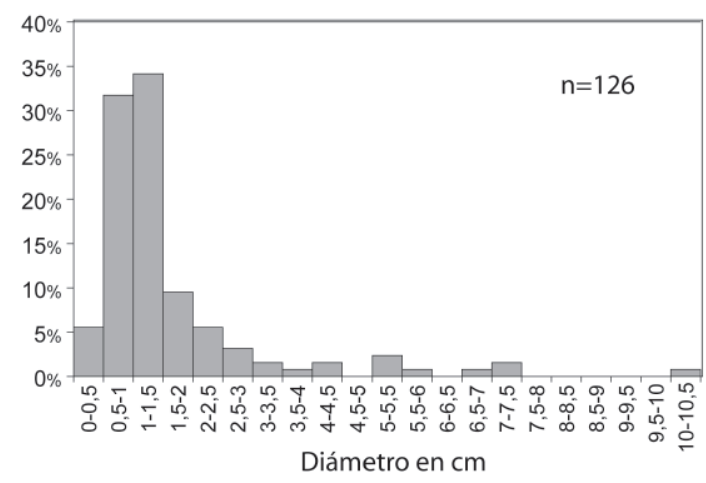

Fig. 14: Abundancia relativa de los diámetros de las raíces fósiles de la isla Catalina ("v", Fig. 2). 
Cuadro 5

Comparación de los parámetros granulométricos y composicionales

\begin{tabular}{ccccccccc}
\hline Duna & Tipo & Med. & Moda & Rango & Asim. & E/R & B/C & Cemento \\
\hline $\mathrm{p}$ & Fósil & 2,7 & 1,7 a 3,7 & -1 a 4,7 & Neg. & 0,3 & 2,3 & Esparita \\
$\mathrm{q}$ & Fósil & 2,1 & 1,7 a 2,7 & 0,2 a 4,2 & Neg. & 0,1 & 1,5 & Esparita \\
$\mathrm{g}$ & Fósil & 1,7 & 1,7 & 0,2 a 3,7 & Neg. & 0,1 & 1,5 & Esparita \\
$\mathrm{t}$ & Fósil & 1,7 & 1,2 a 2,7 & -1 a 4,7 & Neg. & 0,3 & 3,0 & Esparita \\
$\mathrm{b}$ & Fósil & 1,5 & 1,7 a 2,7 & 0,2 a 4,7 & Neg. & 0,3 & 3,0 & Esparita \\
v & Fósil & 1,9 & 1,7 a 2,4 & 0,2 a 4,7 & Neg. & $0,1 / 0,3$ & 2,3 & Micrita \\
y & Actual & 1,2 & 1,2 & $0,2 \mathrm{a} 4,2$ & Sim. & 0,3 & 4,0 & - \\
z & Actual & 1,2 & 1,2 & $0,2 \mathrm{a} 4,2$ & Sim. & 0,3 & 4,0 & - \\
Playa & Actual & 2,0 & 2,2 & -1 a 4,27 & Neg. & $0,3 / 0,5$ & 4,5 & - \\
\hline
\end{tabular}

Med.=Mediana, corresponde con el promedio de medianas de las muestras analizadas para cada duna en unidades $\varphi$. Moda en unidades $\varphi$, en los casos donde se indica un rango se presenta comportamiento polimodal. El rango indica el tamaño de partículas en las muestras analizadas en unidades $\varphi$. Asim.=asimetría de las curvas granulométricas (Neg.=negativa; Sim.=curva simétrica). $\mathrm{E} / \mathrm{R}=$ relación esfericidad/redondez. $\mathrm{B} / \mathrm{C}=$ relación de bioclastos con materiales clásticos. Las dunas "p", " $\mathrm{q}$ " y "g" son de la isla San Pedrito, "t" y "b" de la isla Las Golondrinas, "v" de la isla Catalina “y”, "z" y playa de la isla San José.

La asimetría en las dunas fósiles y sedimentos de playa es negativa, lo que implica que los materiales dominantes son las fracciones más finas de las arenas. La relación entre esfericidad y redondez (E/R) (Cuadro 5) oscila entre 0,1 y 0,3 para todos los materiales, tanto los que han sufrido un proceso de litificación como los inconsolidados.

En cuanto a la composición litológica de las arenas se aprecian diferencias. Las dunas fósiles tienen un importante aporte clástico sobre todo de rocas ígneas sin diferenciar y peridotitas, mientras que las dunas actualmente activas así como los sedimentos de playa presentan un escaso aporte clástico. La relación de bioclastos con materiales clásticos $(\mathrm{B} / \mathrm{C})$ es mucho mayor en las dunas actuales y en los sedimentos de playa que en las dunas fósiles (Cuadro 5). El aumento en el aporte bioclástico evidenciado en las dunas actualmente activas y los sedimentos de playa puede deberse a un mayor desarrollo de la comunidad biológica en los alrededores de las islas, relacionada con el calentamiento ambiental relativo a través de los últimos 16000 años. Además, la disminución del aporte clástico podría ser explicada por el ascenso del nivel del mar en relación con la morfología submarina que presenta la zona.

Durante la formación de las dunas fósiles el nivel del mar mundialmente se encontraba por debajo del nivel actual, entre 100 y 120 m, tomando datos de Barbados y Nueva Guinea (Pinter \& Gardner, 1989; Bard et al., 1990; Miller et al., 2005); esto sin tomar en cuenta el efecto de levantamiento tectónico local en la zona de la península de Santa Elena. Esto permitía que una porción de superficie mayor de rocas con

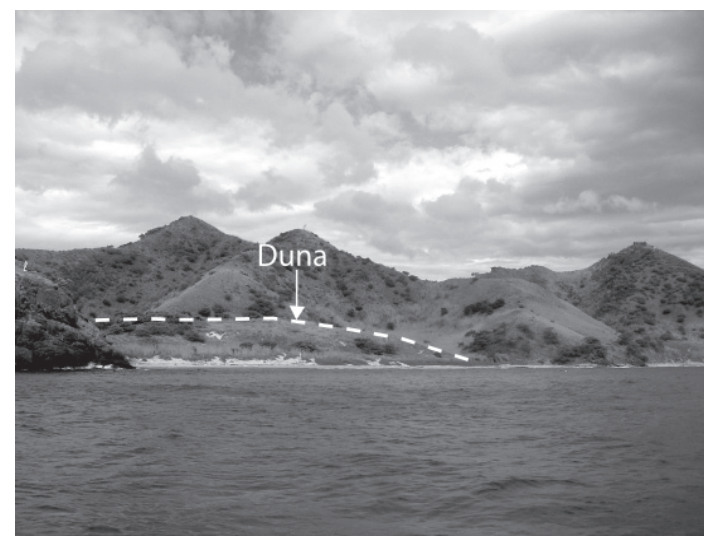

Fig. 15: Duna actual de la isla San José. 
baja pendiente quedara expuesta a la erosión del oleaje, lo que además se encargaba de llevar el sedimento clástico hasta la orilla donde la acción del viento formaba las dunas. Conforme el nivel fue subiendo el área expuesta a la erosión y transporte marino disminuyó drásticamente, y ahora el material erosionado de la zona costera de las islas, cae al fondo en lugar de llegar a la superficie, debido a la fuerte pendiente de fondo marino (Fig. 3).

El proceso de litificación de las dunas fósiles ocurrió sin que estas estuvieran sepultadas, la cementación se produjo como un resultado directo de la acción del aporte dado por el splash marino y la disolución de alguna porción de los fragmentos bioclásticos depositados. El cemento es principalmente esparítico, excepto en las dunas de la isla Santa Catalina donde se da la preservación de raíces de arbustos ("v", Fig. 2), donde el cemento es micrítico. Esta coincidencia nos hace pensar que la actividad biológica jugó un papel predominante en la formación de cemento micrítico que presentan estas dunas.

La presencia de lutitas en los fragmentos de arena de las dunas fósiles, a pesar de que en las islas no afloran rocas lutitas actualmente, es similar al hallazgo de lutitas del Paleoceno en un canto rodado recolectado frente a isla Cocinero (Azéma et al., 1981; Tournon, 1984). Esto puede corresponder a una cobertura sedimentaria de las islas ya erosionada. Las dunas fósiles son muy similares en cuanto a su composición y granulometría, tanto a las dunas activas, como a la playa actual del extremo oriental de isla San José. (Fig. 2).

La relación geográfica de las dunas, así como su distribución y relación a la costa coinciden en que las dunas fósiles fueron dunas costeras, que se formaron por fuertes vientos similares a los alisios que ahora azotan la región. Las dimensiones de las dunas fósiles, algunas con más de $50 \mathrm{~m}$ de longitud y más de $10 \mathrm{~m}$ de espesor, así como la extensión lateral del área de dunas en las islas, que es de $3 \mathrm{~km}$, indican que se formaron en un momento de fuertes vientos y probablemente clima más seco que el actual. Relacionando esto con su edad, que según la datación radiométrica de ${ }^{14} \mathrm{C}$ de los granos que constituyen estas areniscas, da un intervalo para su edad máxima de 15981 y 17132 años $\mathrm{BP}$, lo cual coincide con el evento Heinrich 1 (Clark \& Bartlein, 1995; Robinson et al., 2005), después del período de máxima extensión de la cobertura glaciar de Norte América (Carrara \& Gimser, 1988). También pudieron haberse originado en algún momento antes de la gran decaída de los glaciares en la región, hace unos 12000 años (Lachniet \& Seltzer, 2002), como por ejemplo en períodos regionales muy secos como el ocurrido entre 14500 y 12500 años BP (Polyak et al., 2004).

Es difícil especular sobre la morfología en el momento de formación de las dunas. De acuerdo con los cambios eustáticos, el nivel del mar se encontraba entre 100 y 120 m por debajo del actual (Pinter \& Gardner, 1989; Bard et al., 1990; Miller et al., 2005), el fondo marino entre tierra firme (punta Santa Elena) y las islas está entre 60 y $80 \mathrm{~m}$ de profundidad actualmente (Fig. 3). El levantamiento tectónico de la región no es conocido, pero asumiendo que en la costa pacífica septentrional de Costa Rica el levantamiento casi no sobrepasa los $3 \mathrm{~mm} / \mathrm{año}$ (Denyer et al., 2003), entonces el nivel del mar debe haber estado, al menos, entre 10 y $30 \mathrm{~m}$ por debajo de la línea de costa actual, lo cual corresponde con la plataforma sumergida $20 \mathrm{~m}$ en punta Santa Elena.

El hecho de que solo en las dunas fósiles de la isla Catalina se hallan encontrado moldes de raíces puede indicar que fue la única localidad que pudo mantener una vegetación más grande, probablemente por estar más protegida, debido a la relación entre la dirección del viento con los elementos geográficos, pues precisamente las dunas de esta isla son las más protegidas de los vientos de dirección NW-SE, por su relación con la punta Santa Elena (Fig. 1). Según las estratificaciones cruzadas medidas y graficadas (Fig. 4), se puede inferir que el viento que formó las dunas tuvo direcciones aproximadas a N30W y S69W. 


\section{CONCLUSIONES}

Las dunas fósiles de las islas Murciélago se formaron a finales del Pleistoceno, correspondiendo con el inicio del final de la glaciación Wisconsin, con una edad máxima entre 15981 y 17 132, según datación de ${ }^{14} \mathrm{C}$, correspondiendo con el evento paleo-climático Heinrich 1. Las direcciones de los vientos fueron similares a los actuales, con vectores del NE al SW y del NNW al SSE. En general estas dunas solo tuvieron vegetación más grande en áreas relativamente protegidas, como la isla Catalina. Actualmente las dunas forman un hábitat especial que ha permitido el desarrollo de especies hermatípicas como el arbursto Amphipterygium adstringens.

\section{AGRADECIMIENTOS}

Este trabajo es parte de los proyectos de la Vicerrectoría de Investigación de la Universidad de Costa Rica, \# 113-A5-047 y \# 113-A6-078. El Área de Conservación Guanacaste nos apoyo en el desarrollo de este proyecto, en especial Róger Blanco y Giovanni Bassey. Las granulometrías fueron hechas por Javier Alvarado de la Sección de Geotecnia de la Escuela de Geología (UCR). Las raíces fósiles fueron identificadas por Isabel Carpio del Laboratorio de Maderas de la Facultad de Ingeniería, Universidad de Costa Rica. Jorge Gómez Laurito, Escuela de Biología, Universidad de Costa Rica y Mike Grayum, Missouri Botanical Garden, ayudaron con información sobre la vegetación de las islas.

\section{REFERENCIAS}

AZÉMA, J., GLAÇON, G. \& TOURNON, J., 1981: Nouvelles doneés sur le Paleocene a foraminiferes planctoniques de la bourdure pacifique de Costa Rica (America Centrale).- Bull. Soc. Geol. Fr. $3: 85-88$.
BANICHEVICH, A. \& CASTRO, V., 1999: Imágenes satelitales en la predicción de eventos esporádicos de escala sinóptica: Antecedentes del huracán César como un evento sinóptico.- Top. Meteor. Oceanog. 6(2):1-6.

BARD, E., HAMELIN, B., FARIRBANKS, R.G. \& ZINDLER, 1990: Calibration of the $14 \mathrm{C}$ timescale over the past 30,000 years using mass spectrometric U-Th ages from corals.- Nature, 345: 405-410.

CARRARA, P.E. \& MCGIMSEY, R.G., 1988: Map showing distribution of moraines and extent of glaciers from the mid-19th Century to 1979 in the Mount Jackson area, Glacier National park, Montana.Mapa I-1508-C. US Geol. Sur. Misc. Inv. Ser.

CLARK, P.U. \& BARTLEIN, P.J., 1995: Correlation of late Pleistocene glaciation in the western United States with North Atlantic Heinrich events.- Geology, 23: 483-486.

DAUPHIN, G.L. \& GRAYUM, M. H., 2005: Bryophytes of the Santa Elena Peninsula and Islas Murciélago, Guanacaste, Costa Rica, with special attention to neotropical dry forest habitats.- Lankesteriana, 5: 53-61.

DENGO, G., 1962: Estudio geológico de la región de Guanacaste, Costa Rica.- 112 págs. Instituto Geográfico Nacional, San José.

DENYER, P., MONTERO, W. \& ALVARADO, G.E., 2003: Atlas tectónico de Costa Rica.- 81 págs. Editorial UCR, San José.

FIEDLER, P.C., 2002: The annual cycle and biological effects of the Costa Rica Dome.Deep-Sea Res. 49: 321-338 
FOLK , R.L., 1962: Spectral subdivisions of limestone types. In: HAM , W.G. (ed), Classification of carbonate rocks. Am. Ass. Petrol. Geol. Mem. 1, 62-84.

HARRISON, J.V., 1953: The geology of the Santa Elena peninsula in Costa Rica, Central America.- Seventh Pacific Congress Proceedings, New Zealand, 2, 102-104.

HAUFF, F., HOERNLE, K. \& BOGAARD, P., 2000: Age and geochemistry of basaltic complexes in western Costa Rica: Contributions to the geotectonic evolution of Central America.- Geochemistry, Geophysics, Geosystems $\left(\mathrm{G}^{3}\right)$.- 1(5), doi 10.1029/1999GC000020.

HOFMANN, E.E, BUSALACCHI, A.J. \& O'BRIEN, J.J., 1981: Wind generation of the Costa Rica Dome.- Science, 214: 552-554.

HUGHEN, K.A., BAILLIE, M.G.L., BARD, E., BAYLISS, A., BECK, J.W., BERTRAND, C.J.H., BLACKWELL, P.G., BUCK, C.E., BURR, G.S., CUTLER, K.B., DAMON, P.E., EDWARDS, R.L., FAIRBANKS, R.G., FRIEDRICH, M., GUILDERSON, T.P., KROMER, B., MCCORMAC, F.G., MANNING, S.W., BRONK RAMSEY, C., REIMER, P.J., REIMER, R.W., REMMELE, S., SOUTHON, J.R., STUIVER, M., TALAMO, S., TAYLOR, F.W., VAN DER PLICHT, J. \& WEYHENMEYER, C.E., 2004: Marine04 Marine radiocarbon age calibration, 26 - 0 ka BP.- Radiocarbon, 46: 1059-1086.

MILLER, K.G., KOMINZ, M.A. BROWNING, J.V. WRIGHT, J.D., MOUNTAIN, G.S., KATZ, M.E., SUGARMAN, P.J., CRAMER, B.S., CHRISTIE-BLICK, N. \& PEKAR, S.F., 2005: The Phanerozoic record of global sea-level change.Science, 310: 1293-1298.
PINTER, N. \& GARDNER, T.W., 1989: Construction of a polynomial model of glacio-eustatic fluctuation: estimating paleo-sea levels continuously through time.- Geology, 7: 295-298.

POLYAK, V.J., RASMUSSEN, J.B.T. \& AMERSON, Y., 2004: Prolonged wet period in the southwestern United States through the Younger Dryas.- Geology, 32(1): 5-8.

REITER, F. \& ACS, P., 2002: Tectonics FP.- http:// Tectonicsfp.com/ [consulta: 6 enero 2006.]

ROBINSON, L.F., ADKINS, J.F., KEIGWIN, L.D., SOUTHON, J., FERNANDEZ, D.P., WANG, S-L \& SCHEIRER, D.S., 2005: Radiocarbon variability in the western North Atlantic during the last glaciation.Science, 310: 1469-1473.

STUIVER, M. \& REIMER, P.J., 1993: Extended 14C data base and revised CALIB 3.0 14C Age calibration program.- Radiocarbon, 35(1): 215-230.

STUIVER, M., REIMER, P. J., \& REIMER, R. W. 2005. CALIB 5.0.- [WWW program and documentation]. http://calib.qub. ac.uk/calib/ [consulta: 3 enero 2006].

TOURNON, J., 1984: Magmatismes du Mesozoique á l' actuel en Amerique Centrale: L' exemple de Costa Rica, des ophiolites aux andesites.- 335 págs.. University Pierre and Marie Curie, Paris, [Tesis Ph.D.]

TOURNON, J., 1994: The Santa Elena Peninsula: an ophiolitic nappe and a sedimentary volcanic relative autochthonous.- Profil, $7: 87-96$.

TRASK, P.D., 1930: Mechanical analysis of sediments by centrifuge.- Econ. Geol. 25: 581-599. 